lustitia Socialis. Revista Arbitrada de Ciencias Jurídicas.

Año V. Vol. V. №3. Edición Especial. 2020-III:

Universidad Regional Autónoma de los Andes

Hecho el depósito de Ley: FA2016000064

ISSN: 2542-3371

FUNDACIÓN KOINONIA (F.K). Santa Ana de Coro, Venezuela

José María Beltrán-Ayala; Dalia Maricela Granizo-Navarrete

http://dx.doi.org/10.35381/racii.v5i3.1129

\title{
Centro público especializado para rehabilitación de adolescentes drogodependientes
}

Specialized public center for the rehabilitation of drug-dependent adolescents

\author{
José María Beltrán-Ayala \\ us.josebeltran@uniandes.edu.ec \\ Universidad Regional Autónoma de los Andes, Santo Domingo \\ Ecuador \\ https://orcid.org/0000-0002-3375-2677 \\ Dalia Maricela Granizo-Navarrete \\ us.dalianagranizo@uniandes.edu.ec \\ Universidad Regional Autónoma de los Andes, Santo Domingo \\ Ecuador
}

Recibido: 31 de octubre de 2020

Revisado: 10 de octubre de 2020

Aprobado: 05 de diciembre de 2020

Publicado: 10 de diciembre de 2020 
lustitia Socialis. Revista Arbitrada de Ciencias Jurídicas.

Año V. Vol. V. N³. Edición Especial. 2020-III:

Universidad Regional Autónoma de los Andes

Hecho el depósito de Ley: FA2016000064

ISSN: 2542-3371

FUNDACIÓN KOINONIA (F.K). Santa Ana de Coro, Venezuela

José María Beltrán-Ayala; Dalia Maricela Granizo-Navarrete

\title{
RESUMEN
}

La investigación plantea la necesidad de creación de un centro público especializado en adolescentes drogodependientes en el Cantón Santo Domingo una de las zonas con más incidencia en el consumo de drogas por adolescentes. El objetivo general de la investigación es analizar la importancia de la atención de los adolescentes drogo dependientes en el Cantón Santo Domingo. El desarrollo de la investigación es desde la perspectiva cuantitativa, de tipo descriptiva y se complementó por un diseño documentalbibliográfico. Fueron encuestados 196 personas, se obtuvo la opinión en materia de drogadicción que acontecen en dicha comunidad, para verificar esta realidad se utilizó como herramienta a la encuesta con el fin de obtener de primera mano la información que se tiene del flagelo de las drogas.. Se concluye que es necesario y de forma urgente la creación de un centro que logren no solamente la rehabilitación sino la reinserción social familiar de los adolescentes drogodependientes.

Descriptores: Problemas sociales; adicción; terapia. (Palabras tomadas del tesauro UNESCO).

\begin{abstract}
The research raises the need for the creation of a public center specialized in drugdependent adolescents in the Santo Domingo Canton, one of the areas with the highest incidence of drug use by adolescents. The general objective of the research is to analyze the importance of the care of drug-dependent adolescents in the Santo Domingo Canton. The development of the research is from a quantitative perspective, descriptive and was complemented by a documentary-bibliographic design. 196 people were surveyed, the opinion on drug addiction that occurs in said community was obtained, to verify this reality the survey was used as a tool in order to obtain first-hand information on the scourge of drugs. It is concluded that the creation of a center that achieves not only rehabilitation but also family social reintegration of drug-dependent adolescents is urgently necessary.
\end{abstract}

Descriptors: Social problems; addiction; therapy. (Words taken from the UNESCO thesaurus). 
lustitia Socialis. Revista Arbitrada de Ciencias Jurídicas.

Año V. Vol. V. N³. Edición Especial. 2020-III:

Universidad Regional Autónoma de los Andes

Hecho el depósito de Ley: FA2016000064

ISSN: 2542-3371

FUNDACIÓN KOINONIA (F.K). Santa Ana de Coro, Venezuela

José María Beltrán-Ayala; Dalia Maricela Granizo-Navarrete

\section{INTRODUCCIÓN}

Quizá una de las etapas más complejas que experimenta el ser humano durante su existencia, es la adolescencia, caracterizada por una serie de transformaciones y cambios que se considera vital en su desarrollo y más aún cuando del consumo de drogas se trata. Por eso se ha dicho la adolescencia es hoy una cultura, un modo de vivir, de interpretar el mundo, un conjunto de lenguajes, creencias, mitos, normas y valores. La adolescencia es la interacción de una etapa del sujeto con el mundo, cuya pertenencia no es privativa del adulto. (CONSEP, 2012: 7)

En este sentido es importante conocer que los adolescentes adoptan nuevas responsabilidades, experimentan nuevas formas de hacer las cosas y reclaman con impaciencia su independencia. Comienzan a cuestionarse a sí mismos y a los demás, y a advertir las complejidades y los matices de la vida. También empiezan a pensar sobre conceptos como la verdad y la justicia. (UNICEF, 2002: 2)

En otras palabras, es un periodo de riesgos y amenazas que requieren una respuesta adecuada, no solo de la familia, sino de la sociedad y por supuesto del Estado. Esta condición de vulnerabilidad es relevante para el Estado en su capacidad de reacción y protección de este grupo etario, Ecuador abstrae los nuevos paradigmas y los plasma en la Constitución vigente desde 2008 reconociendo a los "niños, niñas y adolescentes" como sujetos de derecho y parte de los grupos de atención preferente.

Organismos internacionales como la UNICEF, señala que: La guerra y los disturbios civiles, el VIH/SIDA, la industrialización, la urbanización y el creciente desempleo han socavado gravemente la educación y el desarrollo de millones más. A medida que desaparecen las redes sociales tradicionales, la estructura de la familia se remodela ya veces se viene abajo, y la capacidad de los sistemas de apoyo a la familia y la comunidad disminuye. Al ver cómo un mundo pierde seguridad, coherencia y estructuras, los adolescentes se ven abocados con demasiada frecuencia a hacer elecciones difíciles, casi siempre sin nadie que los ayude. (UNICEF, 2002: 4) 
lustitia Socialis. Revista Arbitrada de Ciencias Jurídicas.

Año V. Vol. V. N³. Edición Especial. 2020-III:

Universidad Regional Autónoma de los Andes

Hecho el depósito de Ley: FA2016000064

ISSN: 2542-3371

FUNDACIÓN KOINONIA (F.K). Santa Ana de Coro, Venezuela

José María Beltrán-Ayala; Dalia Maricela Granizo-Navarrete

Efectivamente la proclividad al riesgo (el consumo de alcohol o estupefacientes) al que se enfrenta el adolescente en el Ecuador se incrementa por varios factores, "la carencia de una estructura y apoyo familiar... revelada por la ausencia de uno de los padres o por desarmonía de la pareja, dificultad económica, violencia intrafamiliar, además del sentimiento de abandono, soledad y acogimiento de los padres" (Loor-Briones \& Otros, 2018:135).

El problema de drogas (consumo y adicción) no ha perdido actualidad, siempre ha sido una preocupación para los Estados. El Ecuador reconoce las adicciones como un problema de salud pública, constitucionaliza su deber y obligación en la prevención, tratamiento, control y rehabilitación de adictos y consumidores. Mayor preocupación y atención merecen los adolescentes pues “...cuando las personas más jóvenes comienzan a consumir drogas, mayores son los riesgos para la salud y otras consecuencias" (OEA \& CICAD, 2019, pág. ii). De ahí una vez más la importancia de su vulnerabilidad.

Según el Plan Nacional de Prevención Integral y Control del Fenómeno Socio-Económico de las Drogas 2017-2021, para el año 2016 se informa que en el Ecuador el promedio de inicio del consumo de drogas está entre 14 y 15 años de edad con alto índice de concentración territorial de drogas en las ciudades de Guayaquil, Quito, Manabí y Santo Domingo.

Aquellos índices lejos de disminuir parecen incrementarse cada vez más; por lo que "...el Ecuador requiere actualizar tanto las estrategias, como los objetivos, programas, proyectos y metas, fortalecer los procesos de generación y coordinación de la aplicación de las políticas..." (CONSEP, 2012, pág. 19) con el único propósito de promover y garantizar el desarrollo integral de las y los adolescentes dentro del nuevo modelo de desarrollo denominado "El Buen Vivir".

El fenómeno de la drogodependencia, en 1969 La Organización Mundial refiriéndose a la drogodependencia indica los siguiente es un estado psíquico y en ocasiones también físico, debido a la interacción entre un organismo vivió y una droga que se caracteriza 
lustitia Socialis. Revista Arbitrada de Ciencias Jurídicas.

Año V. Vol. V. N³. Edición Especial. 2020-III:

Universidad Regional Autónoma de los Andes

Hecho el depósito de Ley: FA2016000064

ISSN: 2542-3371

FUNDACIÓN KOINONIA (F.K). Santa Ana de Coro, Venezuela

José María Beltrán-Ayala; Dalia Maricela Granizo-Navarrete

por modificaciones del comportamiento y por otras reacciones, entre las que siempre se encuentra un impulso irreprimible a ingerir de forma continua o periódica con objeto de volver a experimentar sus efectos psíquicos. En el año 2012, La Organización Mundial de la Salud señaló que "muchos países no han reconocido aun la drogodependencia como un problema de salud, y la estigmatización y discriminación asociadas a esos trastornos han sido un gran obstáculo para lograr un tratamiento adecuado" (OMS, 2012). Sin embargo, señala la propia Organización que los beneficios de los tratamientos eficaces de los drogodependientes están siendo reconocidos por los países como ventajosos para sus propias sociedades y economías.

De ahí la importancia de relevar la problemática de la drogodependencia como política fundamental del Estado mediante el diseño de una legislación adecuada a la realidad en convergencia con planes y proyectos (centros de atención y rehabilitación especializados) reales que logren enfrentar y solucionar dicho fenómeno en aras de lograr en los jóvenes el anhelado Buen Vivir.

Como se había señalado inicialmente, la adolescencia requiere de atención prioritaria precisamente por ser una etapa vulnerable y de alto riesgo frente al consumo de drogas. La legislación entendida como parte del componente macro social del fenómeno de la drogodependencia, debe expresar y contemplar la realidad de dicha problemática; la realidad que en el caso de Ecuador "....alerta acerca de aspectos como el consumo de drogas en edades tempranas, el relativo fácil acceso a las mismas y la determinación de las zonas con mayor incidencia del fenómeno socio económico de las drogas." (SETED, 2017: 3). Es en este sentido que la normativa jurídica debe constituir la base y estructura "...encaminadas a reducir el espacio a las drogas y afianzar un adecuado desarrollo del ser humano" (SETED, 2017:42).

Finalmente, en 2015, se promulga la Ley Orgánica de Prevención Integral del Fenómeno Socioeconómico de las drogas y de Regulación y Control del Uso de Sustancias Catalogadas Sujetas a Fiscalización. Esta nueva propuesta legislativa nace a la luz de un nuevo marco constitucional, el denominado "Buen Vivir" que entroniza al ser humano 
lustitia Socialis. Revista Arbitrada de Ciencias Jurídicas.

Año V. Vol. V. N³. Edición Especial. 2020-III:

Universidad Regional Autónoma de los Andes

Hecho el depósito de Ley: FA2016000064

ISSN: 2542-3371

FUNDACIÓN KOINONIA (F.K). Santa Ana de Coro, Venezuela

José María Beltrán-Ayala; Dalia Maricela Granizo-Navarrete

sobre el capital, y cuyo principio fundamental en esta materia es la no criminalización al consumo de sustancias sujetas a fiscalización.

La drogodependencia al amparo de la legislación ecuatoriana actual, a partir de la vigencia de la Constitución del Ecuador (2008) la lógica de los factores de la producción experimenta un dramático cambio, en este sentido el individuo o el ser humano se posiciona sobre el capital y se sitúa "... en el centro del actual estatal" (SETED, 2017: 4) y con relación a las drogas se lo ha distinguido “...como un fenómeno socio económico, lo que demanda una intervención intersectorial e interinstitucional con un enfoque de derechos humanos y de salud pública.

Efectivamente la Norma Suprema (Art. 364) consagra la no criminalización del consumo, considera que las adicciones son un problema de salud pública sujeta medidas de tratamiento y rehabilitación; es decir el eje principal en esta nueva visión constitucional es la "prevención integral", en concordancia así lo establece la Ley Orgánica de Prevención Integral del Fenómeno Socio Económico de las Drogas y de Regulación y Control del Uso de Sustancias Catalogadas Sujetas a Fiscalización, que en uno de sus considerados textualmente cito: la prevención integral del fenómeno socio económico de las drogas debe basarse fundamentalmente en la implementación de políticas públicas en materias de educación y salud, en el marco de una legislación que propicie y facilite la aplicación de dichas políticas, habita cuenta de que es ineludible e inexcusable del Estado el atender Se implementó un enfoque centrado en el sujeto y en el respeto a los derechos humanos, brindando atención a las personas con consumo problemático, con procesos terapéuticos integrales, dirigidos al individuo, familia y sociedad para la disminución de los años asociados al consumo problemático de drogas.(SETED, 2017: 8)

El Código Orgánico Integral Penal, conocido por sus siglas como COIP, entró en vigencia el 10 de febrero de 2014, mismo que rige hasta la actualidad, (concebido dentro del nuevo modelo del Sumak Kawsai) en el Art. 220 último inciso, atendiendo el principio constitucional de la no criminalización del consumo, dispone: "la tenencia y posesión de 
lustitia Socialis. Revista Arbitrada de Ciencias Jurídicas.

Año V. Vol. V. N³. Edición Especial. 2020-III:

Universidad Regional Autónoma de los Andes

Hecho el depósito de Ley: FA2016000064

ISSN: 2542-3371

FUNDACIÓN KOINONIA (F.K). Santa Ana de Coro, Venezuela

José María Beltrán-Ayala; Dalia Maricela Granizo-Navarrete

sustancias estupefacientes o psicotrópicas para uso o consumo personal en las cantidades establecidas por la normativa correspondiente, no será punible".

Se debe resaltar que en materia de menores infractores la ley regula las inconductas es el Código de la Niñez y la Adolescencia.

El objetivo general de la presente investigación es analizar la importancia de la atención de los adolescentes drogo dependientes en el Cantón Santo Domingo.

\section{METODOLOGÍA}

El desarrollo de la investigación es desde la perspectiva cuantitativa, de tipo descriptiva y se complementó por un diseño documental-bibliográfico, (Parela-Stracuzzi \& MartinsPestana, 2012) permitiendo la recogida de información extraída de los diferentes textos jurídicos y organismos multilaterales, abordando el método analítico enfocándose en el estudio de aspectos particulares, examinados en el análisis de contenido escrito, mediante lo analítico-sintético (Bernal-Torres, 2006). Fueron encuestados 196 personas del sector Santo Domingo de se obtuvo la opinión en materia de drogadicción que acontecen en dicha comunidad, para verificar esta realidad se utilizó como herramienta a la encuesta con el fin de obtener de primera mano la información que se tiene en cuanto al flagelo de las drogas.

\section{RESULTADOS Y DISCUSIÓN}

Siendo Santo Domingo una zona de consumo y ante la ausencia de lugares públicos especializados para el tratamiento de menores drogodependencia que permitan su rehabilitación y por ende la reinserción familiar y social, es menester conocer y discutir los resultados de las consecuencias que arrojaron la muestra obtenida para este efecto. 
lustitia Socialis. Revista Arbitrada de Ciencias Jurídicas.

Año V. Vol. V. №3. Edición Especial. 2020-III:

Universidad Regional Autónoma de los Andes

Hecho el depósito de Ley: FA2016000064

ISSN: 2542-3371

FUNDACIÓN KOINONIA (F.K). Santa Ana de Coro, Venezuela

José María Beltrán-Ayala; Dalia Maricela Granizo-Navarrete

1.- Consumo de drogas en adolescentes en el cantón Santo Domingo.

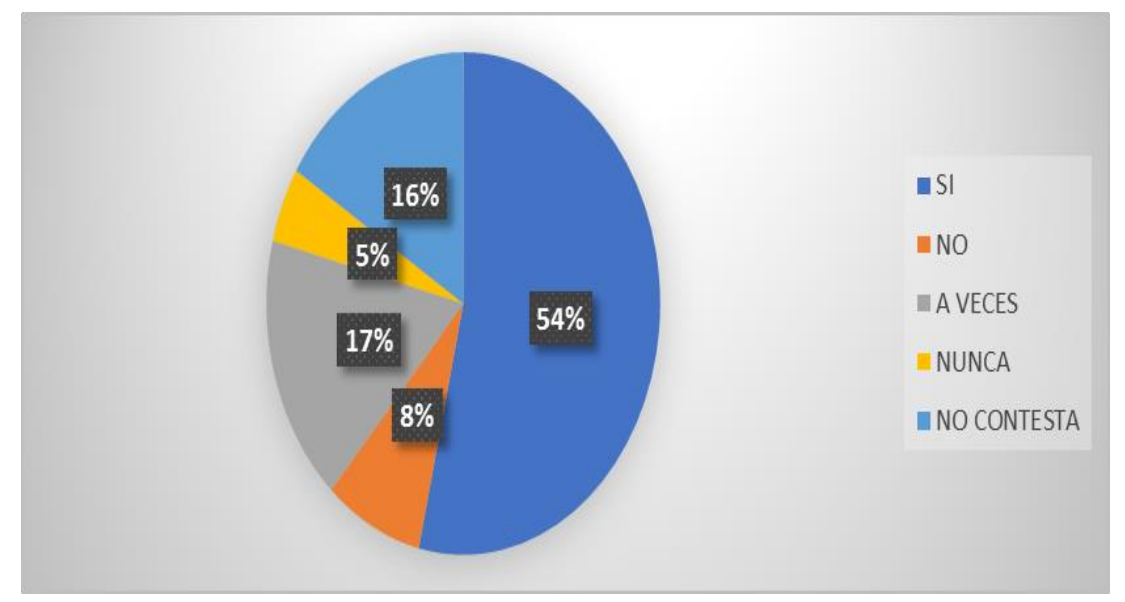

Gráfico 1.

Elaboración por: Autores (2021).

Fuente: Resultados de la encuesta.

La pregunta encaminada a saber si el encuestado alguna vez consumió algún tipo de droga, revelan de alguna manera las cifras oficiales que señalan al cantón Santo Domingo como una zona de alto consumo del Ecuador. De los 196 encuestados, 133 personas aceptan haber consumido alguna droga, 20 personas responden que no, 43 personas señalan que a veces recurren al consumo de drogas, un mínimo de 12 personas que niegan haber consumido y finalmente 40 personas no contestan. 
lustitia Socialis. Revista Arbitrada de Ciencias Jurídicas.

Año V. Vol. V. №3. Edición Especial. 2020-III:

Universidad Regional Autónoma de los Andes

Hecho el depósito de Ley: FA2016000064

ISSN: 2542-3371

FUNDACIÓN KOINONIA (F.K). Santa Ana de Coro, Venezuela

José María Beltrán-Ayala; Dalia Maricela Granizo-Navarrete

2.- Conocimiento de las instituciones que receptan denuncias sobre consumo o venta de drogas.

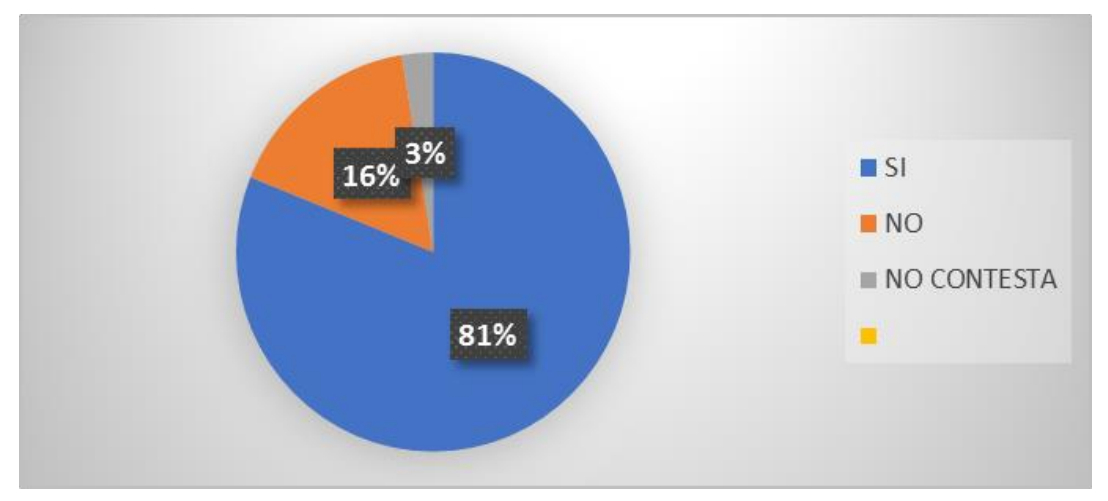

Gráfico 2.

Elaboración por: Autores (2021).

Fuente: Resultados de la encuesta.

De los 196 encuestados, 159 personas no conocen cuáles son las instituciones que receptan las denuncias sobre el consumo o venta de drogas/instituciones como la Fiscalía y la Policía) frente a 32 que dicen si conocer a donde dirigirse en estos casos. 5 de los encuestados no contestan. Es necesario que es Estado a través de sus principales instituciones como por ejemplo la Fiscalía, la Policía Nacional y el Consejo de la Judicatura socialicen su rol dentro de la sociedad a nivel de la comunidad con incidencia mayoritaria en un territorio donde es más proclive el uso y consumo de drogas. 
lustitia Socialis. Revista Arbitrada de Ciencias Jurídicas.

Año V. Vol. V. №3. Edición Especial. 2020-III:

Universidad Regional Autónoma de los Andes

Hecho el depósito de Ley: FA2016000064

ISSN: 2542-3371

FUNDACIÓN KOINONIA (F.K). Santa Ana de Coro, Venezuela

José María Beltrán-Ayala; Dalia Maricela Granizo-Navarrete

3.- Conocimiento del procedimiento en denuncias sobre consumo o vena de drogas.

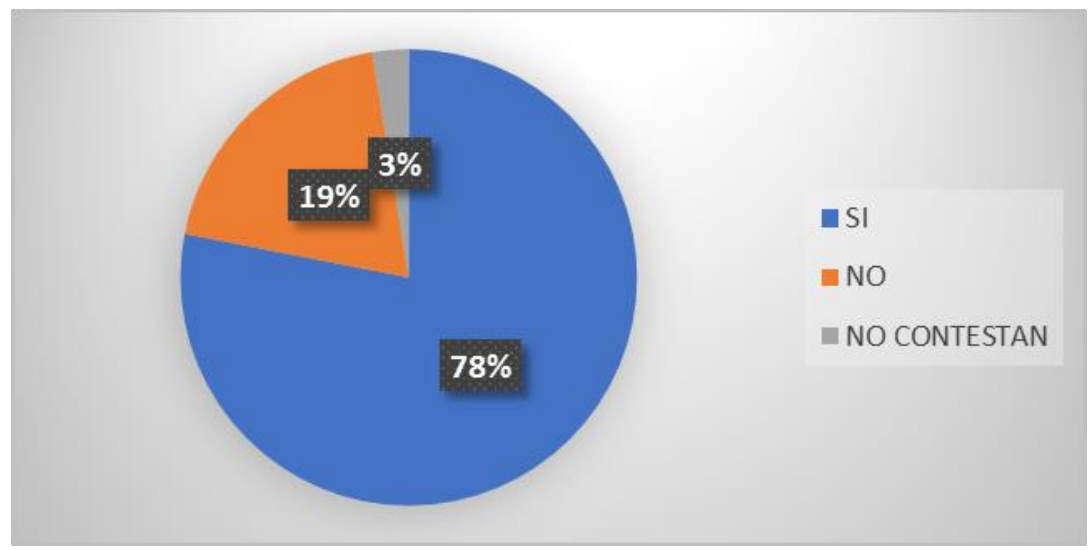

Gráfico 3.

Elaboración por: Autores (2021).

Fuente: Resultados de la encuesta.

Frente a la pregunta si conocen el procedimiento que deben seguir para presentar una denuncia por consumo o venta de drogas, es lógico el resultado, pues como se refleja en el cuadro anterior (Cuadro No. 3), si las personas no saben a dónde dirigirse para presentar una denuncia sobre drogas, mucho menos conocen su procedimiento. Efectivamente 153 personas desconocen, frente a 38 que dicen saber el procedimiento y 5 encuestados no contestan. 
lustitia Socialis. Revista Arbitrada de Ciencias Jurídicas.

Año V. Vol. V. №3. Edición Especial. 2020-III:

Universidad Regional Autónoma de los Andes

Hecho el depósito de Ley: FA2016000064

ISSN: 2542-3371

FUNDACIÓN KOINONIA (F.K). Santa Ana de Coro, Venezuela

José María Beltrán-Ayala; Dalia Maricela Granizo-Navarrete

4.-Criminalización del uso y consumo de drogas por adolescentes.

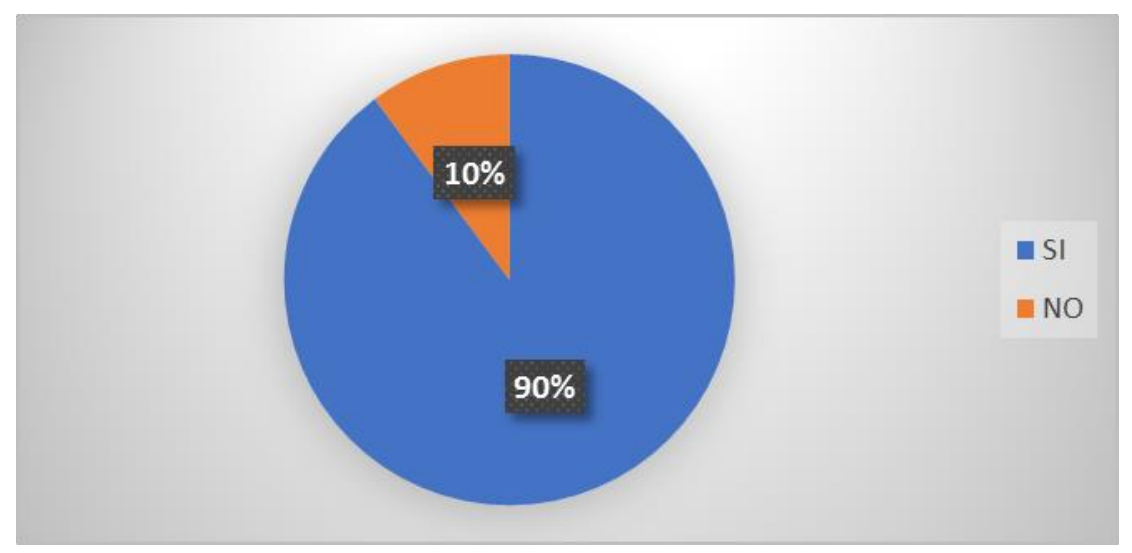

Gráfico 4.

Elaboración por: Autores (2021).

Fuente: Resultados de la encuesta.

De una muestra de 196 personas encuestadas, 176 personas contestan negativamente a la pregunta si considerarían al adolescente drogodependiente como delincuente, mientras que tan solo 20 responden afirmativamente. Posiblemente la nueva política pública en el Ecuador que reconoce el problema de las drogas como un fenómeno socioeconómico dejando atrás la política represiva sea la razón por la que actualmente en el cantón Santo Domingo no se criminalice a los adolescentes drogodependientes. 
lustitia Socialis. Revista Arbitrada de Ciencias Jurídicas.

Año V. Vol. V. №3. Edición Especial. 2020-III:

Universidad Regional Autónoma de los Andes

Hecho el depósito de Ley: FA2016000064 ISSN: 2542-3371

FUNDACIÓN KOINONIA (F.K). Santa Ana de Coro, Venezuela

José María Beltrán-Ayala; Dalia Maricela Granizo-Navarrete

5.- Causas de la Drogodependencia en adolescentes.

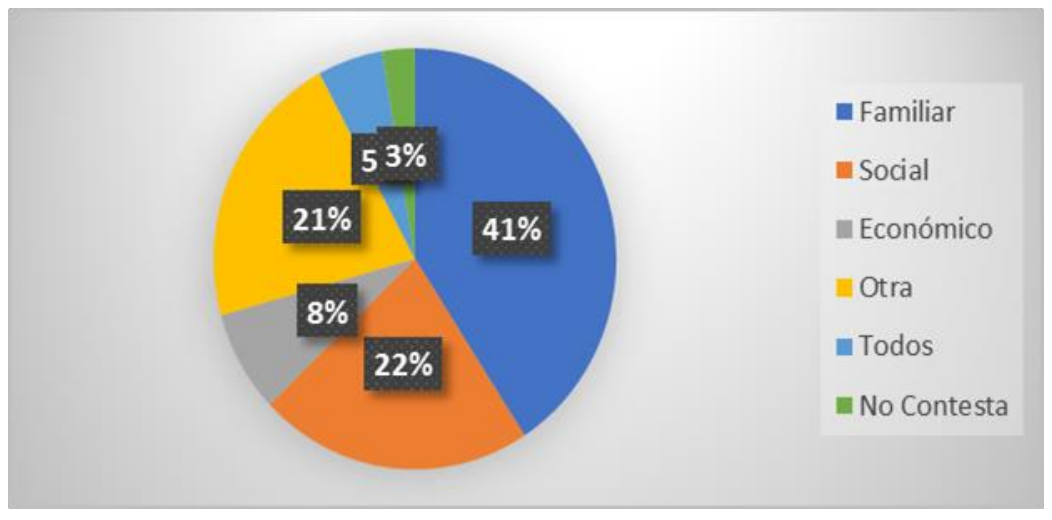

Gráfico 5.

Elaboración por: Autores (2021).

Fuente: Resultados de la encuesta.

Como observamos en la gráfica, 78 personas contestan que son causas de tipo familiar (divorcio, violencia intrafamiliar, maltrato, etc.) que conducen a los jóvenes adolescentes al consumo y uso de drogas; 42 personas contestan que son factores sociales (escuela, comunidad, iglesia, Estado, entre otros) que provocan la drogodependencia; 15 personas responden que es la economía la que provoca este fenómeno (el desempleo, la crisis económica, etc.); 41 personas señalan que son otras causas (hobbies, desastres, psicológicos); 10 personas señalan que son todos los factores (familiar, social, económico) que obligan a los jóvenes adolescentes al uso y consumo de drogas; y, finalmente 10 encuestados no contestan. es decir que la mayoría de personas encuestadas concuerdan con que una de las principales causas de la drogodependencia en adolescentes es por problemas de tipo familiar. 
lustitia Socialis. Revista Arbitrada de Ciencias Jurídicas.

Año V. Vol. V. №3. Edición Especial. 2020-III:

Universidad Regional Autónoma de los Andes

Hecho el depósito de Ley: FA2016000064

ISSN: 2542-3371

FUNDACIÓN KOINONIA (F.K). Santa Ana de Coro, Venezuela

José María Beltrán-Ayala; Dalia Maricela Granizo-Navarrete

6.-Necesidad de un centro público especializado en adolescentes con drogodependencia.

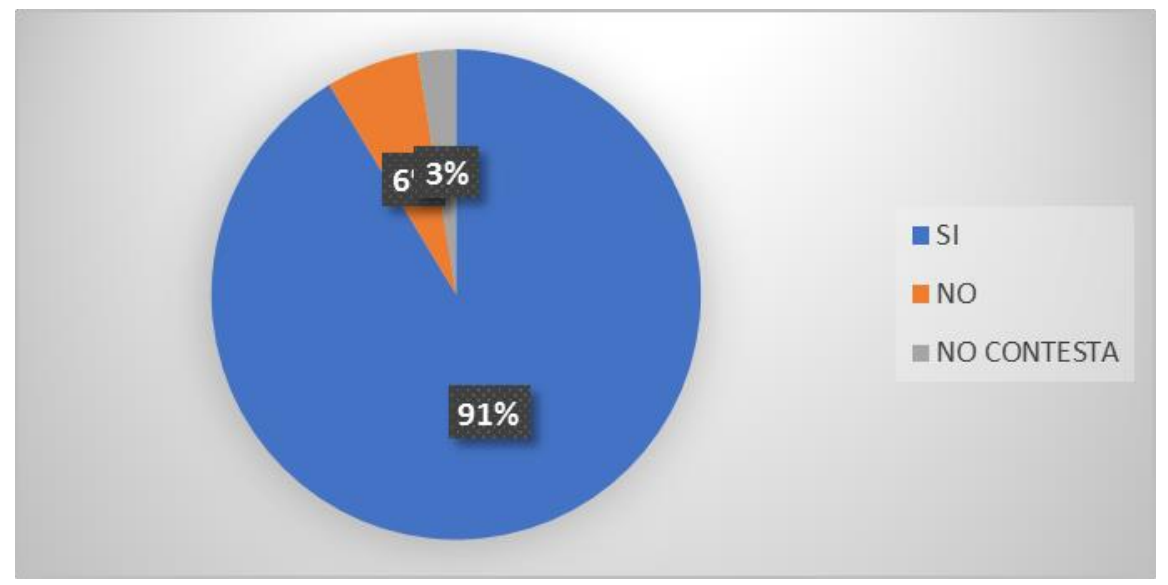

Gráfico 6.

Elaboración por: Autores (2021).

Fuente: Resultados de la encuesta.

Frente a la pregunta si considera necesario crear en Santo Domingo de los Colorados un centro público especializado que atienda la drogodependencia de los adolescentes, de los 196 encuestados, 179 personas responden afirmativamente, 12 personas consideran que no y 5 no contestan. por lo tanto, dentro de esta pregunta se puede establecer que es sumamente importante y necesario la creación de este tipo de centro en el cantón. 
lustitia Socialis. Revista Arbitrada de Ciencias Jurídicas.

Año V. Vol. V. №3. Edición Especial. 2020-III:

Universidad Regional Autónoma de los Andes

Hecho el depósito de Ley: FA2016000064

ISSN: 2542-3371

FUNDACIÓN KOINONIA (F.K). Santa Ana de Coro, Venezuela

José María Beltrán-Ayala; Dalia Maricela Granizo-Navarrete

7.- Sometimiento a tratamiento de recuperación de adolescentes drogodependientes en un centro público especializado.

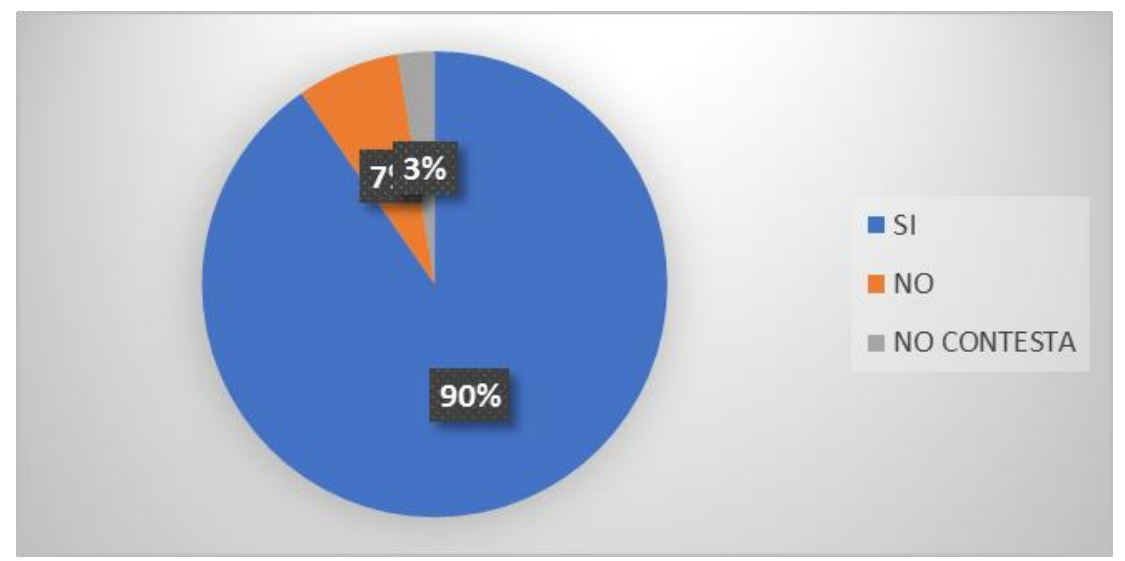

Gráfico 7.

Elaboración por: Autores (2021).

Fuente: Resultados de la encuesta.

Frente a la pregunta si pondría a un adolescente consumir drogas someterse a un tratamiento de recuperación en un centro público especializado, de los 196 encuestados, 177 personas afirman que sí lo harían, 14 personas responden negativamente y 5 de los encuestados no contestan. esto afirma que no solamente urge la creación de un centro público de tratamiento y rehabilitación en Santo Domingo, sino que adicionalmente la sociedad se involucra en dicho fenómeno como se lo plantea en este trabajo de investigación. 
Iustitia Socialis. Revista Arbitrada de Ciencias Jurídicas.

Año V. Vol. V. N³. Edición Especial. 2020-III:

Universidad Regional Autónoma de los Andes

Hecho el depósito de Ley: FA2016000064

ISSN: 2542-3371

FUNDACIÓN KOINONIA (F.K). Santa Ana de Coro, Venezuela

José María Beltrán-Ayala; Dalia Maricela Granizo-Navarrete

\section{CONCLUSIÓN}

Las drogas se han constituido en un fenómeno que no deja de preocupar tanto más cuando quien las usa y las consume con mayor frecuencia son los adolescentes, cuya adicción no solamente tiene implicación en la salud, sino que también incide en la esfera social y económica.

Ecuador recoge aquella preocupación y la plasma en su Constitución reconociendo el fenómeno de las drogas como un problema de salud pública a la par titulariza a los adolescentes como parte del grupo de vulnerables y por ende su atención prioritaria en lo público y privado.

Santo Domingo se ha convertido en una zona de alto consumo de drogas por parte de los adolescentes, quienes, por causas familiares, económicas, sociales entre otras, recurren a su uso y consumo, aquello tiene implicación a nivel individual, social y estatal. Ante la ausencia de un establecimiento público especializado en Santo Domingo que atienda de forma holística la drogodependencia de los menores adolescentes, es necesario y de forma urgente la creación de uno de estos centros que logren no solamente la rehabilitación sino la reinserción social familiar de los adolescentes drogodependientes.

\section{FINANCIAMIENTO}

No monetario.

\section{AGRADECIMIENTO}

A la Universidad Regional Autónoma de los Andes; por motivar el desarrollo de la Investigación. 
lustitia Socialis. Revista Arbitrada de Ciencias Jurídicas.

Año V. Vol. V. N³. Edición Especial. 2020-III:

Universidad Regional Autónoma de los Andes

Hecho el depósito de Ley: FA2016000064 ISSN: 2542-3371

FUNDACIÓN KOINONIA (F.K). Santa Ana de Coro, Venezuela

José María Beltrán-Ayala; Dalia Maricela Granizo-Navarrete

\section{REFERENCIAS CONSULTADAS}

Asamblea Nacional Constituyente de la República del Ecuador, (2008). Constitución de la República del Ecuador. Montecristi. Registro Oficial 449 de 20-oct-2008. Recuperado de https://n9.cl/sia

Asamblea Nacional de la República del Ecuador. (2014). Código Orgánico Integral Penal [Comprehensive Organic Criminal Code]. Recuperado de https://n9.cl/g6sc

Asamblea Nacional del Ecuador (2015) Ley Orgánica de Prevención Integral Fenómeno Socio Económico Drogas. Registro Oficial Suplemento 615 de 26-oct-2015

Asamblea Nacional del Ecuador. (2009). Ley Reformatoria al Código de la Niñez y Adolescencia. [Reform Law to the Childhood and Adolescence Code]. Quito: Asamblea Nacional del Ecuador. Obtenido de https://n9.cl/ul23

Bernal-Torres, C. A. (2006). Metodología de la investigación. [Research methodology] Recuperado de https://n9.cl/8txy

Congreso Nacional del Ecuador. (1970). Código Civil. Título preliminar. [Civil Code] Quito, Pichincha, Ecuador: Registro Oficial No. 104

Consejo Nacional de Control de Sustancias Estupefacientes y Psicotrópicas (2012) Plan Nacional de Prevención Integral de Drogas 2012-2013. [National Comprehensive Drug Prevention Plan 2012-2013] Recuperado de: https://n9.cl/b5e3m

Loor-Briones, W., Hidalgo-Hidalgo, H., Macías-Alvarado, J., García-Noy, E. \& ScrichVázquez, A. (2018). Causas de las adicciones en adolescentes y jóvenes en Ecuador. [Causes of addiction in adolescents and young people in Ecuador] Revista Archivo Médico de Camagüey, 22(2), 130-138. Recuperado de: https://n9.cl/3mk25

OEA, \& CICAD. (2018). Informe sobre el Consumo de Drogas en las Américas 2019. [Report on Drug Use in the Americas 2019]. SEXAGÉSIMO CUARTO PERÍODO ORDINARIO DE SESIONES Del 19 al 21 de noviembre de 2018 Washington, D.C., EE.UU. recuperado de: https://n9.cl/bx4m5

Organización Mundial de la Salud (2012) Hay que mejorar el acceso de los drogodependientes a la atención sanitaria. [Drug addicts' access to health care needs to be improved] Recuperado de: https://n9.cl/b8pli 
lustitia Socialis. Revista Arbitrada de Ciencias Jurídicas.

Año V. Vol. V. №3. Edición Especial. 2020-III:

Universidad Regional Autónoma de los Andes

Hecho el depósito de Ley: FA2016000064

ISSN: 2542-3371

FUNDACIÓN KOINONIA (F.K). Santa Ana de Coro, Venezuela

José María Beltrán-Ayala; Dalia Maricela Granizo-Navarrete

Palella Stracuzzi, S. \& Martins Pestana, F. (2012). Metodología de la investigación cuantitativa. [Quantitative research methodology]. Fondo editorial de la Universidad Pedagógica Libertador. Caracas, Venezuela.

Secretaría Técnica de Prevención Integral de Drogas (2012) Plan Nacional de Prevención Integral de Drogas 2012-2013. [National Comprehensive Drug Prevention Plan 2012-2013]. Recuperado de: https://n9.cl/b5e3m

UNICEF (2002). Adolescencia una etapa fundamental. [ Adolescence a fundamental stage]. Recuperado de: https://n9.cl/tbkti

(C2020 por los autores. Este artículo es de acceso abierto y distribuido según los términos y condiciones de la licencia Creative Commons Atribución-NoComercial-Compartirlgual 4.0 Internacional (CC BY-NC-SA 4.0) (https://creativecommons.org/licenses/by-nc-sa/4.0/). 